\section{Unsicherheit und Ungewissheit}

\author{
Falko von Ameln · Telse Iwers ${ }^{1}$
}

Angenommen: 20. Oktober 2021 / Online publiziert: 24. November 2021

(c) Der/die Autor(en) 2021

Die Rede von der „VUKA-Welt“ prägt seit Jahren die Diskurse in Management und Organisationswissenschaften. Spätestens mit der Corona-Krise ist die Wahrnehmung von Ungewissheit und Unsicherheit in allen gesellschaftlichen Kontexten angekommen. Natürlich ist das Thema trotz seiner Aktualität keineswegs neu und wird seit vielen Jahren aus verschiedenen disziplinären Perspektiven beleuchtet

Beispielsweise wird die Bedeutung epistemischer Ungewissheit in kommunikationswissenschaftlichen Kontexten u. a. vor dem Hintergrund zunehmender Digitalisierung und damit verbundener digitaler Verunsicherung in der ,,posttruth era" thematisiert (van der Bles et al. 2020). In pädagogischen Kontexten werden mit Blick auf Bildungsinstitutionen und Bildungsdynamiken vor dem Hintergrund systemtheoretischer Konzepte Fragen des Umgangs mit Ungewissheit und der Entwicklung von Ungewissheitstoleranz in technologiedefizitären Interaktionssituationen gewendet.

Im Hinblick auf individuelle Entwicklungen werden einerseits Fragen von Ermöglichung, Entscheidungsvielfalt und Verfügbarkeit gegenüber Unsicherheit, Ergebnisoffenheit und Ambiguität im Kontext von Lebensbewältigung virulent. Andererseits werden aus entwicklungspsychologischer wie auch kognitionspsychologischer Perspektive Auswirkungen von Lernen und Entwicklung in von Ungewissheit oder Unsicherheit gekennzeichneten Situationen modelliert und evaluiert.

Das vorliegende Themenheft der Zeitschrift GIO wendet sich der zunehmenden Virulenz von Ungewissheit und Unsicherheit im organisationalen Zusammenhang zu. Ausgehend von Untersuchungen im Zusammenhang mit individuellen Entscheidungsprozessen wurden in der Organisationspsychologie Umgangsweisen von nicht-trivialen Systemen in zunehmend komplexen Umwelten betrachtet. Schon vor 100 Jahren schlug Frank Hyneman Knight eine Klassifikation von Unsicherheit und Risiko vor (Boeckelmann und

Telse Iwers

Telse.Iwers@uni-hamburg.de

1 Fakultät für Erziehungswissenschaft, Universität Hamburg, Hamburg, Deutschland
Mildner 2011). Eine Situation, in der die Eintretenswahrscheinlichkeit eines bestimmten Ereignisses logisch oder empirisch bestimmt werden kann, ist von Risiko geprägt: Die Wahrscheinlichkeit, dass eine Mitspielerin beim Poker mit sieben Karten ein Full House hat, beträgt rechnerisch 2,6\%. Die Wahrscheinlichkeit, dass die Spielerin bei einem Einsatz blufft, lässt sich dagegen nicht statistisch ermitteln. Diese Form der Unsicherheit nennt Knight Ungewissheit (true uncertainty). John Maynard Keynes wies darauf hin, dass viele Entscheidungssituationen in Organisationen von radikaler Unsicherheit (radical uncertainty) bestimmt sind, da organisationale Umwelten in vielerlei Hinsicht zu volatil sind und das Handeln der beteiligten Akteure zu viele Freiheitsgrade aufweist, um gesicherte Prognosen zuzulassen.

Die Forschung zu komplexen Systemen hat gezeigt, dass komplexe Systeme angesichts einer Vielzahl zu berücksichtigender Variablen und für außenstehende Beobachter/ -innen intransparenter interner Rückkopplungen nicht nur schwer zu prognostizieren sind, sondern aufgrund ihrer Eigendynamik Emergenzphänomene produzieren und daher grundsätzlich unvorhersehbar sind. Organisationen haben es mit zahlreichen solcher nicht-trivialer Systeme (Heinz von Foerster) zu tun - vom Verhalten der einzelnen Mitarbeiterin über die eigene Organisationskultur bis hin zu hochkomplexen und volatilen ökonomischen und politischen Umwelten.

Radikale Unsicherheit kann verschiedene Quellen haben - bei Magnani und Zucchella (2018) sowie bei Samsami et al. (2015) findet sich ein aktueller Überblick über die Forschung zu den verschiedenen Dimensionen:

- Unsicherheit über den Ausgang von Umweltentwicklungen (z. B. Komplexität, Dynamik, Marktunsicherheit, technologische Unsicherheit)

- Unsicherheit über die Handlungen anderer Akteure (z. B. Unsicherheit durch Konkurrenz, mangelnde Vertrautheit, kulturelle Unsicherheit, Unsicherheit in der Kommunikation)

- Unsicherheit durch Nichtwissen (z. B. unvollständige Information) 
Als Treiber der Zunahme von Unsicherheit in den organisationalen Umwelten wurden in den letzten Jahren u.a. die Digitalisierung, der Klimawandel und die damit verbundenen staatlichen und gesellschaftlichen Veränderungen (z. B. steigende Umweltauflagen, sinkende Akzeptanz fossiler Technologien für Mobilität und Energiegewinnung) oder der Fachkräftemangel diskutiert. Krisenphänomene wie die Corona-Pandemie tragen zu einer Steigerung von Unsicherheit bei. Wenn die Diagnose von Ulrich Beck (1990, 2008) zutrifft, stellt der Umgang mit Unsicherheit aber eine Daueraufgabe moderner Gesellschaften dar, insbesondere auch, da Strategien der „Komplexitätsreduktion“ (Döring-Seipel und Lantermann 2015, S. 2). mit ihren teilweise einschränkenden Wirkungen für Organisationen Platz greifen.

Dies gilt in besonderem Maße für Organisationen. Sie sind kontinuierlich mit dem Versuch beschäftigt, trotz Unsicherheit Stabilität zu erlangen, ihren Bestand zu sichern und ihre Ziele zu verwirklichen. So ist z.B. das strategische Management, die „Königsdisziplin“ des Managements, zentral damit beschäftigt, den Kontingenzen einer unsicheren Zukunft mit Planung zu begegnen und damit Unsicherheit in Risiko zu verwandeln. Dass das strategische Management keineswegs Sicherheit, sondern allenfalls notwendige Scheinsicherheiten produziert, hat schon Henry Mintzberg (1994) eindrücklich gezeigt. Aus organisationssoziologischer Sicht trägt es - wie viele andere Managementpraktiken auch - eher den Charakter einer ritualistischen, magischen Praktik, die der Unsicherheitsabsorption dient, auch wenn sie wenig zum Erfolg beiträgt.

Dass die Bewältigung von Unsicherheit eine Aufgabe ist, die an Bedeutung gewinnt, zeigt sich auch am Bedeutungszuwachs, den Stressbewältigung in der Personal- und Organisationsentwicklung der letzten Jahrzehnte erfahren hat. Die wachsenden Unsicherheiten haben einen hohen Veränderungsdruck und - damit verbunden - eine bedeutende Belastungssteigerung für die Mitarbeitenden mit sich gebracht. Ambiguitätstoleranz gilt als Schlüsselkompetenz für die Gestaltung der Organisationen der Zukunft.

Das vorliegende Themenheft der Zeitschrift GIO beleuchtet organisationale Herausforderungen im Umgang mit Unsicherheit und Ungewissheit aus verschiedenen Blickwinkeln, die sich aus Psychologie, Soziologie, Pädagogik und Sozialer Arbeit speisen. Damit verwirklicht dieses Heft, wie wir meinen, den Anspruch der Zeitschrift, von einem organisationspsychologischen Standpunkt aus interdisziplinäre Perspektiven einzubinden, in besonderer Weise.

Falko von Ameln gibt einen Überblick über die Herausforderungen, mit denen Führen und Entscheiden unter Unsicherheit konfrontiert sind. Der Autor plädiert dafür, den sozialen Kontext des Entscheidens stärker zu berücksichtigen als dies in der klassischen psychologischen Entscheidungstheorie bislang der Fall ist. Am Beispiel der deutschen Corona-Politik wird u.a. aufgezeigt, welche Spannungsfelder sich durch die Notwendigkeit der Akzeptanzsicherung angesichts widersprüchlicher Erwartungen auftun und welche strukturellen und kulturellen Rahmenbedingungen seitens der Organisation für Führen und Entscheiden unter Unsicherheit funktional bzw. dysfunktional sind.

Auch Jennifer Sparr zeigt in ihrem Beitrag ,,Vom Paradox zur Resilienz in der Krise: Ein Modell für erfolgreiches Krisenmanagement" anhand der Pandemie auf, dass Krisenmanagement vor allem im Ausbalancieren von Widerspruchsfeldern besteht. Den Schlüssel hierzu sieht die Autorin in einem ,,paradoxen Mindset“ der Führungskräfte, das diese zu einer positiven und annehmenden Haltung gegenüber paradoxen Spannungsfeldern befähigt. Auf Basis eines solchen Mindsets, so die Autorin, könne die Konstruktion geteilten Sinns ebenso gelingen wie Chancen für eine resilientere Organisationsgestaltung wahrgenommen und durch schrittweises Lernen realisierbar würden.

Martin Elbe und Sibylle Peters reflektieren unter der Überschrift „Neue Räume - neue Rollen? Ungewissheit im Kontext der Temporären Organisation“, welche Unsicherheiten arbeitsweltliche Entwicklungen für Beschäftigte und Führungskräfte produzieren und mit welchen Veränderungen auf der Ebene beruflicher Identitäten diese Unsicherheiten einhergehen. Die Autor/-innen schlagen dabei einen weiten Bogen von der Auflösung räumlicher Bezüge und zeitlicher Kontinuitäten im Verlauf der beruflichen Entwicklung über die Identitätsarbeit von Beschäftigten bis hin zu changierenden Rollenkonzepten.

Stephanie Rascher gibt in ihrem Beitrag „Führung in Zeiten der Ungewissheit - die Rolle einer konstruktiven Fehlermanagement- und Vertrauenskultur als Ressource in Krisenzeiten" einen Überblick über Aspekte, die Führungskräfte gestalten müssen, um Krisensituationen kompetent zu meistern. Auf der Ebene der Mitarbeitenden beleuchtet Rascher die Faktoren Ambiguitätstoleranz, Situationsbewusstsein und Resilienz, auf der Teamebene geteiltes Lageverständnis, Kooperation und Entscheidungsfindung sowie psychologische Sicherheit und auf der Organisationsebene flexible Kultur, Gerechtigkeitskultur und Lernkultur.

Anke Redecker plädiert in ihrem Beitrag „Ungewissheit als Fremdheitserfahrung - Zum Umgang mit Diversität in der lernenden Organisation“ auf Basis der Phänomenologie der Fremdheit für eine Erweiterung anerkennungstheoretischer Annahmen um die Anerkennung der, eigenen Fremdheit durch Selbstentzogenheit " und damit verbundene Projektionen. Reflexionen pädagogischen Handelns in Ungewissheitssituationen können diese Projektionen sicht- und lösbar werden lassen.

Michael Heinlein und Norbert Huchler geht es in ihrem Beitrag „Digitalisierung als Lösung für Ungewissheit in Organisationen? Neue Wege der sozio-technischen Erwartbarkeit unsicherer Zukünfte" darum, in den Spannungsfeldern von Formalität und Informalität sowie von Mensch 
und Technik neue Gestaltungsmöglichkeiten für die Bearbeitung von Unsicherheit zu entwickeln. Ihr Konzept der prospektiven Organisation versucht Chancen von technologiebasierter Steuerung und neuen Arbeitsformen zu einer hybriden Mensch-Technik-Organisation zu verbinden. Die Autoren illustrieren dieses Konzept anhand von zwei Fallbeispielen aus kleinen und mittelständischen Unternehmen (KMU).

Sonja Kristine Ötting, Lisa Masjutin und Günter W. Maier beschreiben in ihrem Beitrag ,The future of leadership How is leadership in small and medium-sized enterprises going to change?" die durch Digitalisierung ausgelöste zunehmende Unsicherheit von KMU im Hinblick auf veränderte Anforderungen an Führung. In einer qualitativen Studie zeigen sie, dass die befragten Führenden Veränderungen in den Organisationsstrukturen erwarten, auf die mit zunehmender Agilität und Diversität reagiert wird.

Bernd Eckstein und Judith Muster analysieren in ihrem Beitrag ,Postbürokratie und die agile Unsicherheitsabsorption in Interaktionen“ auf Basis der Theorie sozialer Systeme den Umgang mit postbürokratisch bedingt neu entstehenden Unsicherheiten. Deren Bewältigung ist mit agilen Managementkonzepten wie z.B. Scrum und Holacracy erreichbar, wobei neue Formalisierungsbedarfe entstehen, die auf Interdependenzen in Interaktionen fokussieren.

Sezen Merve Yilmaz \& Telse A. Iwers diskutieren in ihrem Beitrag ,Entwicklung eines reflexionsorientierten Umgangs mit heterogenitätsbedingter Ungewissheit" die Entstehung von Stereotypisierungen und subjektiven Theorien des Otherings durch kognitive Schließungen in von Ungewissheit geprägten Interaktionssituationen. Anhand eines transkulturellen Begegnungsprojektes werden Dekonstruktionsgelegenheiten aufgezeigt.

Herbert Effinger rekonstruiert in seinem Beitrag ,Lost in Uncertainty? Sozial entscheiden und organisieren in der Sozialwirtschaft" die Entwicklung, die der soziale Sektor im politischen Kontext in den vergangenen Jahren genommen hat und die laut Effinger zahlreiche Ungewissheiten mit sich gebracht hat. Der im Zuge dieser Entwicklung entstandene „Wohlfahrtsmix“, so der Autor, begünstigt Vermeidungsverhalten und organisiertes Misstrauen, erschwert Vertrauensbildung, Arbeitsbündnisse und Kooperationsbeziehungen und begünstigt Stress. Die Diskussion dieser Thesen führt zu einem Plädoyer für eine Forschung, die diese Ungewissheitsfaktoren in ihrem funktionalen Wert für die Akteure sieht und näher ergründet.

Karola Cafantaris untersucht in dem Beitrag „Eine ethnographische Annäherung an die Erforschung von Ungewissheit in hybriden Lernsettings" den Umgang mit pädagogischer Ungewissheit in hybriden Lernsettings der Erwachsenenbildung. Sie zeigt anhand eines ethnographischen Beobachtungsprotokolls auf, welche Kommunika- tionsformate auf den Umgang mit Ungewissheit in dem gewählten Setting verweisen.

Im offenen Teil geht Paul Reinbachers Beitrag „Führung als System: Ein handlungstheoretischer Bezugsrahmen zur Konsolidierung des klassischen Leadership-Diskurses mit T. Parsons" von der These aus, dass die zeitgenössische Führungsforschung und Führungslehre wenig integriert sind, weil ihnen ein theoretischer Bezugsrahmen fehlt. Der Autor schlägt Parsons AGIL-Schema als einen solchen möglichen Bezugsrahmen vor und verortet die wichtigsten theoretischen Ansätze zum Thema Führung grob in diesem Modell.

Auch Rudolf Wimmer beschäftigt sich mit dem Thema Führung, nimmt aber unter der Überschrift „Führung von und in Familienunternehmen" eine theoriebezogene Standortbestimmung zu einem bedeutsamen (Wimmer ordnet 70-90\% der weltweiten Unternehmen diesem Typus zu), aber nach vor wenig erforschten Thema vor. Nach einer Rekonstruktion der wesentlichen Besonderheiten dieses Unternehmenstyps stellt Wimmer drei dominante Ansätze der internationalen Familienunternehmensforschung vor: die Principal-Agent-Theorie, die Stewardship-Theorie und den Social-Emotional-Wealth-Ansatz. Wenn Wimmer diesen Forschungslinien ein systemtheoretisches Verständnis von Führung als Reaktion auf organisationsspezifische Unsicherheitsquellen entgegensetzt, schlägt dies den Bogen wieder zurück zum Thema dieses Heftes.

Wir wünschen allen Leserinnen und Lesern eine erkenntnisreiche Lektüre!

Funding Open Access funding enabled and organized by Projekt DEAL.

Open Access Dieser Artikel wird unter der Creative Commons Namensnennung 4.0 International Lizenz veröffentlicht, welche die Nutzung, Vervielfältigung, Bearbeitung, Verbreitung und Wiedergabe in jeglichem Medium und Format erlaubt, sofern Sie den/die ursprünglichen Autor(en) und die Quelle ordnungsgemäß nennen, einen Link zur Creative Commons Lizenz beifügen und angeben, ob Änderungen vorgenommen wurden.

Die in diesem Artikel enthaltenen Bilder und sonstiges Drittmaterial unterliegen ebenfalls der genannten Creative Commons Lizenz, sofern sich aus der Abbildungslegende nichts anderes ergibt. Sofern das betreffende Material nicht unter der genannten Creative Commons Lizenz steht und die betreffende Handlung nicht nach gesetzlichen Vorschriften erlaubt ist, ist für die oben aufgeführten Weiterverwendungen des Materials die Einwilligung des jeweiligen Rechteinhabers einzuholen.

Weitere Details zur Lizenz entnehmen Sie bitte der Lizenzinformation auf http://creativecommons.org/licenses/by/4.0/deed.de.

\section{Literatur}

Beck, U. (1990). Risikogesellschaft: Auf dem Weg in eine andere Moderne. Frankfurt a.M.: Suhrkamp.

Beck, U. (2008). Weltrisikogesellschaft: Auf der Suche nach der verlorenen Sicherheit. Frankfurt a.M.: Suhrkamp. 
van der Bles, A.M., van der Linden, S., Freeman, A.L. J., \& Spiegelhalter, D. J. (2020). The effects of communicating uncertainty on public trust in facts and numbers. Proc Natl Acad Sci U S A. https://doi.org/10.1073/pnas.1913678117.

Boeckelmann, L., \& Mildner, S.-A. (2011). Unsicherheit, Ungewissheit, Risiko. SWP Zeitschriftenschau 2. https://www.swp-berlin. org/fileadmin/contents/products/zeitschriftenschau/2011zs02_ bkm_mdn_ks.pdf. Zugegriffen: 23. Mai 2021.

Döring-Seipel, E., \& Lantermann, E.-D. (2015). Komplexitätsmanagement. Berlin, Heidelberg, New York: Springer. https://doi.org/10. 1007/978-3-658-08658-9_1.

Magnani, G., \& Zucchella, A. (2018). Uncertainty in entrepreneurship and management studies: A systematic literature review. International Journal of Business and Management, 13(3), 98-133.

Mintzberg, H. (1994). The rise and fall of strategic planning. New York: Prentice-Hall

Samsami, F., Hosseini, S.H.K., Kordnaeij, A., \& Azar, A. (2015). Managing environmental uncertainty: From conceptual review to strategic management point of view. International Journal of Business and Management, 10(7), 215-229.

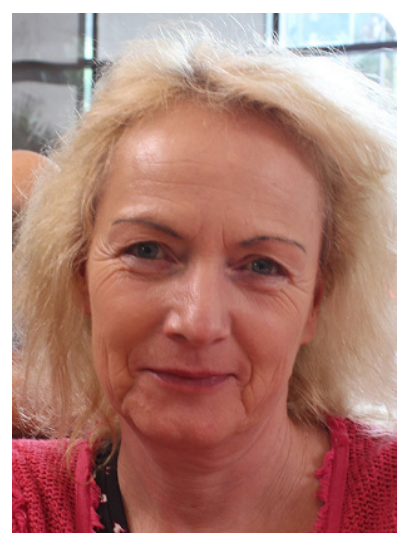

Dr. MHEd. Telse Iwers ist Professorin für Erziehungswissenschaft unter besonderer Berücksichtigung der Pädagogischen Psychologie, Universität Hamburg. Bis 2020 war sie Sprecherin der Kommission Pädagogik und Humanistische Psychologie der Sektion 13 der DGfE. Sie ist Editor in Chief der Zeitschrift „Gruppe. Interaktion. Organisation" und in verschiedenen Projekten zur Achtsamkeitsentwicklung und zur Internationalisierung der Lehrerbildung aktiv. Forschungsschwerpunkte: Reflexion, Beratung und Supervision im Kontext pädagogischer Professionalisierung zur Entwicklung von Ungewissheitstoleranz und Achtsamkeit durch Introvision; pädagogische Sensibilisierung im Umgang mit Heterogenität und Migration. 